Route choice, travel time variability, and rational inattention

Jiang, Gege; Fosgerau, Mogens; Lo, Hong K.

Published in:

Transportation Research Procedia

DOI:

10.1016/j.trpro.2019.05.026

Publication date:

2019

Document version

Publisher's PDF, also known as Version of record

Document license:

CC BY-NC-ND

Citation for published version (APA):

Jiang, G., Fosgerau, M., \& Lo, H. K. (2019). Route choice, travel time variability, and rational inattention. Transportation Research Procedia, 38, 482-502. https://doi.org/10.1016/j.trpro.2019.05.026 


\title{
Route choice, travel time variability, and rational inattention
}

\author{
Gege Jiang ${ }^{\mathrm{a}, *}$, Mogens Fosgerau ${ }^{\mathrm{b}}$, Hong K. Lo ${ }^{\mathrm{a}}$ \\ ${ }^{a}$ Hong Kong University of Science and Technology, Hong Kong,999077 \\ ${ }^{b}$ University of Copenhagen, Copenhagen,1165, Denmark
}

\begin{abstract}
This paper sets up a rational inattention model for the route choice problem in a stochastic network where travelers face random travel time. Previous research has assumed that travelers incorporate all provided information without effort. This study assumes that information is costly and that travelers rationally choose how much information to acquire prior to choosing route. We begin with a single traveler and then extend the model to heterogeneous travelers where rationally inattentive user equilibrium (RIUE) is achieved. From the perspective of a single traveler, more information always reduces the impact of travel time variability and increases the probability of choosing a less costly route. However, in RIUE, more information may reduce the social welfare in some scenarios.
\end{abstract}

(C) 2019 The Authors. Published by Elsevier B.V.

Peer-review under responsibility of the scientific committee of the 23rd International Symposium on Transportation and Traffic Theory.

Keywords: rational inattention, travel time variability, imperfect information, information strategy, discrete choice

\section{Introduction}

The route choice behavior of travelers has been a research topic for many years. Early deterministic network equilibrium models (Wardrop, 1952) deal with constant link capacity and travel demand. In reality, travelers commonly face uncertain travel time with randomness resulting from either the demand side or the supply side in congested transport networks. Stochastic equilibrium models have been proposed to capture network uncertainty, such as the travel time budget (TTB) model and the mean-excess travel time (METT) (e.g., Daganzo and Sheffi, 1977; Xu et al., 2011; Gao et al., 2010; Lo et al., 2006; Chen et al., 2011).

To reduce the impact of travel time variability, real life travelers may take advantage of many information sources, such as radio or TV broadcasting, websites or real time traffic information apps. Recently, researchers have become increasingly interested in studying the impact of information on travel behavior (e.g., Paz and Peeta, 2009; Bifulco et al., 2016). Ettema and Timmermans (2006) proposed a model that analytically captures the cost caused by bias

\footnotetext{
${ }^{*}$ Corresponding author. Tel.: +852-64809254

E-mail address: gjiang@ust.hk
} 
in prior knowledge about travel time distribution and they found that information can reduce the bias. Based on the quality of information, the benefits of travel time information vary. Arnott et al. (1991) asked whether information provision is beneficial for reducing congestion in a route choice problem. They set up a network of two routes with variable capacity and investigated the case with no information, perfect information and imperfect information respectively and concluded that imperfect information may be harmful for the system performance. Similarly, Lindsey et al. (2014) analyzed the impact of pre-trip information about traffic conditions. They also inspected cases where information may be welfare-reducing if free-flow travel costs differ appreciably. Gao (2012) extended the work in a time-dependent stochastic network and investigated travelers' adaptive behavior to information by formulating the equilibrium as a fixed-point problem. Other works used agent-based simulation (Wahle et al., 2002), conducted empirical experiments (Lu et al., 2014; Avineri and Prashker, 2006), modeled route and mode choice simultaneously (Liu et al., 2017), etc.

In recent years, the emerging technology of smart phone, mapping software, and social media provides us with a large amount of traffic information. Travellers have easy access to the real-time information through Google Map, Waze, Radio and many other sources. It is natural to assume that the effort related to information is neglectable. It is the reason that most of the previous mentioned research assumes that travelers effortlessly incorporate all the provided information into their decisions. That assumption implies that every traveler has perfect information of traffic condition with the help of the real-time information sources. To test the validity of the assumption, we conducted an online survey of of 237 drivers belonging to distinct groups of age, occupation, gender, driving frequency and get the following results. We asked the the questions about their frequency of driving, frequency of using real-time information, and the sources of real-time information. From Fig.1, we can see that the percentage of people who always using the real-time information is only $29 \%$. More than half of the drivers do not often refer to real-time information while driving. From Fig.2, the main source of the real-time information is the mapping softwares, followed by radio. The social media such as facebook, twitter, waze are only referred by a small proportion of drivers.

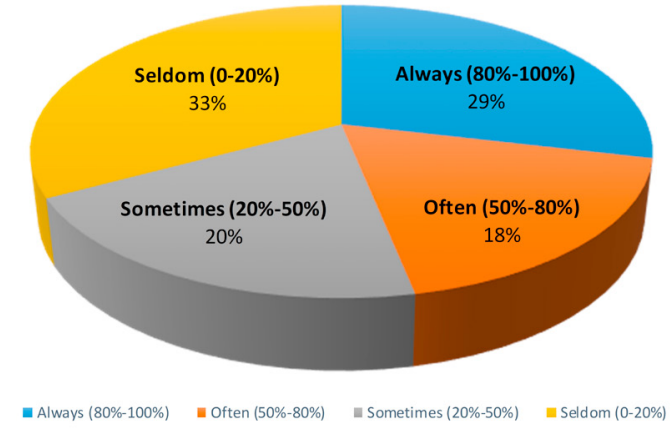

Fig. 1. Frequency of using the real-time information while driving

We further analyzed the percentage of using the real-time information through different age groups. The results are shown in Fig.3. We can see that the percentage of the people who always use real-time information decreases with age. In contrast, the percentage of people seldom using real-time information increases with age. We can learn from the survey that the young people are more prone or have easier access to real-time information. However, even among the young people, there is still a big proportion who seldom use real-time information.

In summary, the survey implies that there is still some barrier for travelers to use real-time information, which might be the psychological characteristics, behavioral preferences, unwillingness to use new technology or etc. The limited human information-processing capacity induces travelers to only consider information selectively (Hogarth, 1987). In reality, travelers are unlikely to make use of all available information as that would require considerable effort, which may outweigh the potential gains from travel cost reduction. Therefore, it is reasonable to assume that the amount of information to be acquired or taken into account is a choice in itself. This choice depends on the cost and benefits of information acquisition. For different groups of people, the information cost may distinguishes between each other. The cost does not only indicates the monetary cost, but also all the possible factors that discourage the travelers to use the real-time information. 


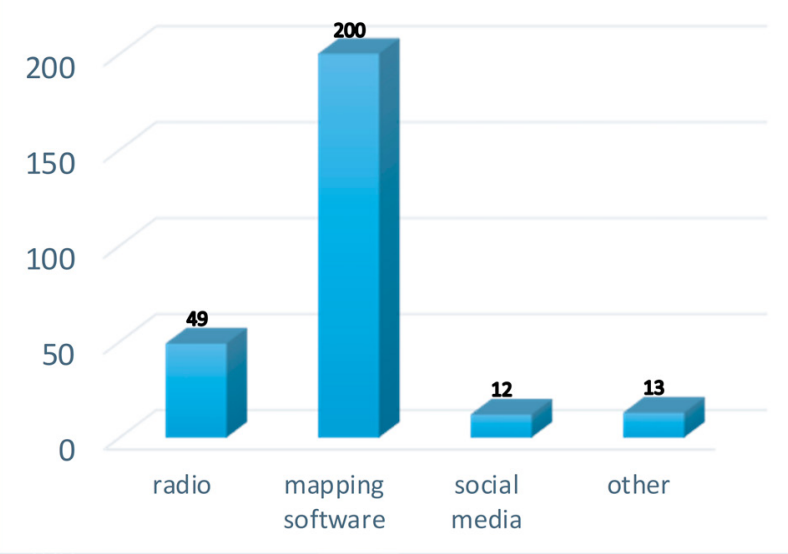

Fig. 2. Sources of the real-time information

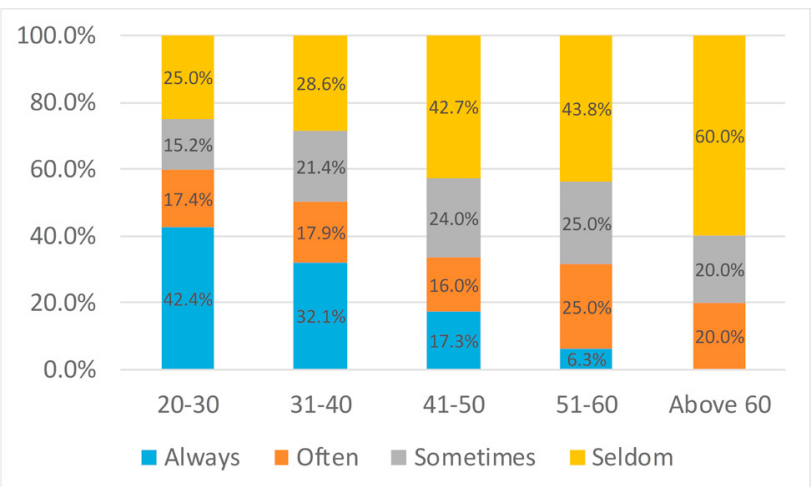

Fig. 3. The relationship between age and the frequency of using real-time information

Recognizing our limited information capacity, Sims (2003) and Sims et al. (2010) proposed a theoretical framework that takes the choice of information acquisition into account, which is called the rational inattention model in micro-economic theory. People choose optimally how much information to take into account, balancing the cost of information against the gains from better informed expectations. In this framework, people will neglect some information with poor reward. The rational inattention model makes it possible to study analytically how the cost of information processing influences peoples' behavior under uncertainty. The model has generated much attention in economics. Recently, Matejka and McKay (2015) showed that in a discrete choice setting, the optimal information strategy leads to a multinomial logit model, when information is measured using the Shannon entropy. This is a very attractive result as it provides a bounded rationality foundation for the logit model. Following their work, Caplin et al. (2016) derived the necessary and sufficient conditions for the solution of rational inattention model. Martin (2017) and Matějka (2015) adapted this model to strategic pricing where the consumers have imperfect information. Fosgerau et al. (2017) extended the framework to provide a bounded rationality foundation for any ARUM discrete choice model.

Application of the rational inattention model requires the prior information of agents to be specified. This is a lesser issue in a transportation setting such as in Fosgerau and Jiang (2019), who considered the departure time choice for a rationally inattentive traveler, since the prior may be taken as the raw unconditional distribution of travel times.

Under the framework of the rational inattention discrete choice model, this study investigates travelers' route choice behavior with imperfect knowledge about traffic conditions. The paper makes the following contributions. First, the rational inattention model is introduced to the route choice problem. Unlike previous research where the information is exogenously given, this study enables the travelers to choose their information strategies. Second, our 
model can be viewed as a generalized model for studying network equilibrium with imperfect information, where the situation with no information and perfect information are incorporated as extreme cases. Third, consideration sets arise endogenously in this model; they are not ad hoc fixes to a computational problem of the modeler, but behavior that arises because travelers economize on information. Fourth, this paper shows that information may have a large impact on travelers' consideration set and on their probability of choosing the optimal route. We begin with the route choice problem for a single traveler and then extend our model to the context of user equilibrium. This study demonstrates that the rational inattention model can be used to study the collective behavior under equilibrium formulated as a fixed-point problem. Fifth, the concept of rationally inattentive user equilibrium (RIUE) is proposed to model the user equilibrium in network with heterogeneous information cost. The impact of imperfect information is examined from both the perspective of the individual traveler and the whole transportation system.

The structure of the paper is as follows. Section 2 introduces the rational inattention model. We begin with a brief introduction of the general idea and properties of the rational inattention model. The relationship between the signal, route choice and the travel time cost is explained. The total individual travel cost is formulated as the sum of the expected travel time cost and the information cost. Section 3 explores the impact of information on the route choice of a single traveler facing travel time variability. In this setting, the travel time of each link is a random. The traveler chooses an information strategy to minimize the total travel cost. His choice will not affect the travel time distribution of the network. Section 4 formulates rationally inattentive user equilibrium (RIUE) in a stochastic network with heterogeneous travelers. In a numerical example, we investigated the impact of information from both the perspective of social welfare and the travelers. Section 5 concludes the paper.

\section{The rational inattention model}

We will set up a rational inattention model for the traveler's route choice problem from the perspective of a single traveler. This section lays out the generic model, which is then specialized to the route choice setting in Section 3.

The state of the traffic system is random and belongs to a finite set of possible states $\Omega$, where $\omega \in \Omega$ denotes a generic state. This is a convenient simplification; it is not restrictive as any continuous travel time distribution may be approximated arbitrarily well by a sufficiently fine discretization. The traveler has prior knowledge about the traffic states encoded in a probability distribution across states $p(\omega)$. The action available to the traveler is the choice of route and the set of available routes is denoted $A$. The cost function $c: A \times \Omega \rightarrow \mathbb{R}$ assigns a cost to each action in each state. The random state $\omega$ is unobserved by travelers prior to their choice of action.

The traveler's decision problem has two stages. In the first stage, each traveler chooses an information strategy in the form of a signal $Z$ : a random variable that carries some information about the state. A perfectly informative signal is a one-to-one function of the state, while an uninformative signal is independent of the state. The choice of signal $Z$ is associated with a cost that increases with the informativeness of the signal; we make this explicit below. The traveler trades off his information cost against his expected travel cost in the second stage. The information cost includes any effort made by the travelers and not just monetary costs. Although the information is becoming more available due to the development of information technologies, some travelers still do not take the time or effort to search for information. As we discuss below, we will use the Shannon entropy to measure the amount of information.

The signal is described by the posterior belief, and the posterior distribution of the state is conditional on the value of the signal $p(\Omega \mid Z)$. The posterior belief is related to the prior through Bayes' law:

$$
p(\omega)=\sum_{z \in Z} p(\omega \mid z) p(z)
$$

In the second stage, the traveler observes a realization of the random signal. With that information, he ${ }^{1}$ chooses the optimal route, his action, based on his posterior belief. Since the signal is random, that makes also the action a random variable.

An important property of the rational inattention model is that it is not necessary to consider the signal explicitly. The signal may be thought of as being implicit in the choice. The reason is the following. If different values of the

\footnotetext{
${ }^{1}$ Gender of representative person chosen at random.
} 
signal lead to the same action, then the signal is inefficient: if the traveler chose an alternative signal that did not distinguish between such values then the alternative signal would have lower information cost, but would lead to the same actions. This argument shows that the traveler would choose a signal where different values of the signal correspond to different actions. Then it is no loss of generality to simply identify the signal with the action. The signal may be taken just as indicating which action to take. It is an important advantage of the rational inattention model that the traveler's choice of information strategy can be considered as implicit in the dependence of choices on the state. Then restrictive assumptions about particular information technologies can be avoided. This is important as in reality there are many different information sources that travelers can use.

The information strategy can then be represented by the conditional probability matrix $P(A \mid \Omega)$. Write $p(a \mid \omega)=$ $P(A=a \mid \Omega=\omega)$ as short-hand for the conditional probability of choosing action $a$ in state $\omega$ and let

$$
p(a)=P(A=a)=\sum_{\omega \in \Omega} p(a \mid \omega) p(\omega)
$$

be the unconditional probability of choosing action $a$. This matrix summarizes how the traveler incorporates information about the state in his action.

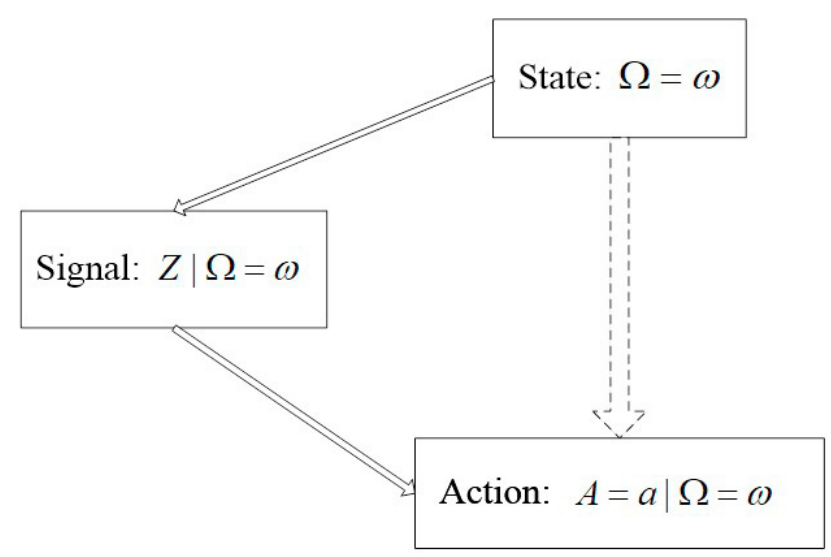

Fig. 4. Decision process

Fig. 4 illustrates the relationship between state $\Omega$, signal $Z$ and action $A$. The solid arrows represent the underlying decision-making process. The traveler first choose an information strategy. According to the information strategy, he receives the signal $Z$ containing information about the state and then he chooses his action. As explained, the signal is implicit represented in the joint distribution of state and action. The dashed line represents the simplified representation of the information strategy.

We now discuss the cost of information. The simplest measure of information is the mutual Shannon information, which is defined in terms of the Shannon entropy. If a discrete random variable $Z$ has the probability distribution $p()$, then its Shannon entropy $H(Z)=-E[\log p(Z)]$ captures the amount of information encoded in $Z$.

The mutual Shannon information between random variables $A$ and $\Omega$ is by definition

$$
\begin{aligned}
I(A ; \Omega) & =H(A)-H(A \mid \Omega) \\
& =\sum_{a \in A} p(a) \log \frac{1}{p(a)}-\sum_{a \in A, \omega \in \Omega} p(a, \omega) \log \frac{1}{p(a \mid \omega)} \\
& =-\sum_{a \in A} p(a) \log p(a)+\sum_{\omega \in \Omega, a \in A} p(a \mid \omega) p(\omega) \log p(a \mid \omega)
\end{aligned}
$$


The traveler then chooses information strategy $P(A \mid \Omega)$ to minimize his total expected cost, which is the sum of his expected travel cost and his information cost,

$$
\Lambda(P)=E(c(A, \Omega))+\lambda I(A, \Omega),
$$

The first term is the expected travel cost with the set of state contingent choice probabilities. The second term is the total information cost, where $\lambda$ is the cost per unit of information and $I(A, \Omega)$ is the amount of information about the state comprised in the action. The information cost involves not only monetary cost but also the time and effort spent by the travelers in acquiring and processing the information. The emergence of smartphones and services such as Google Maps have reduced the cost of information but it still requires effort from travelers to incorporate this information.

Before going into the details of the traveler's solution to this optimization problem, we can make some initial observations about the two extreme cases of complete and no information. The case of complete information arises if information is free, $\lambda=0$. In that case, the traveler is able to choose the shortest path in every state, i.e. $a^{*}(\omega)=$ $\operatorname{argmin}_{a} c(a \mid \omega)$.

In the polar case when information is infinitely expensive, the traveler will choose not to obtain any information therefore his information cost $I(A, \Omega)$ is zero, which implies that his action is independent of the state. In other words, the traveler makes the same route choice every day. In that case, $a^{*}(\omega)$ does not depend on the state $\omega$ and the traveler chooses route to minimize the expected travel cost across all states, i.e. $a^{*}=\operatorname{argmin}_{a} E[c(a \mid \omega)]$. The uninformed traveler is not able to accommodate variability in travel times by adapting his route choice.

\section{Route choice for a rationally inattentive traveler}

The mathematical derivation of the solution and properties of the rational inattention model have been studied extensively (e.g., Matejka and McKay, 2015; Caplin et al., 2016). This paper is the first to cast the route choice problem in terms of rational inattention. In this section, some general properties of the rational inattention model are applied the route choice problem of a single traveler without considering user equilibrium. We examine the impact of information on the size of the consideration set and the distribution of choices across routes. The traveler's total expected cost can be written out in terms of the conditional probabilities $p(a \mid \omega)$ as

$$
\begin{aligned}
& \Lambda(P)=\sum_{\omega \in \Omega} \sum_{a \in A} p(a \mid \omega) p(\omega) c(a \mid \omega)+\lambda\left[-\sum_{a \in A} p(a) \log p(a)+\sum_{\omega \in \Omega, a \in A} p(a \mid \omega) p(\omega) \log p(a \mid \omega)\right] \\
& c(a \mid \omega)=\sum_{i} c(i \mid \omega) \epsilon_{i}^{a} \\
& \sum_{a} p(a \mid \omega)=1 \\
& \sum_{a} p(a \mid \omega) \geq 0
\end{aligned}
$$

where $c(i \mid \omega)$ and $c(a \mid \omega)$ are the link travel time and the path travel time respectively. Let the link-path incidence matrix have generic element $\epsilon_{i}^{a}$, which is 1 if link $i$ is on path $a$ and 0 otherwise. The traveler minimizes his total expected cost subject to (5)-(7). The first order condition for (4) yields

$$
p(a \mid \omega)=\frac{\exp (-c(a \mid \omega) / \lambda) p(a)}{\sum_{a^{\prime}} \exp (-c(a \mid \omega) / \lambda) p\left(a^{\prime}\right)}
$$

The existence of the solution of (8) can be proved using the Brouwer fixed-point theorem. However, if there are identical options in the consideration sets, the solution might not be unique, we will show the case of non-uniqueness in the numerical examples. It is immediately clear that (8) has the form of a logit model, except that the exponentiated negative costs are weighted by the unconditional probabilities $p(a)$. The unconditional probabilities $p(a)$ depend in 
turn on the conditional probabilities according to (2). Finding the conditional and unconditional probabilities then entails solving a fixed point problem, where the presence of the weights $p(a)$ has the effect of amplifying variations in the state-dependent costs $c(a \mid \omega)$. The conditional probability $p(a \mid \omega)$ tends to be large if the unconditional probability $p(a)$ is large, which happens if route $a$ is generally attractive across states.

In cases where $p(a)$ is constant across the choice set, then these terms cancel out of expression for the conditional choice probabilities and the standard logit model results. Otherwise, if the number of states and actions are sufficiently small, then it is possible to derive the solution analytically. In general, however, the solution to (8) and (2) can only be found numerically.

An important thing to note is that some unconditional probabilities may be zero. Clearly, if $p(a)=0$ then also all the conditional probabilities $p(a \mid \omega)$ are zero. Such an option is not chosen in any state. Zero probabilities occur naturally and often in the rational inattention discrete choice model due to the reinforcement impact of the unconditional probabilities on the conditional probabilities in (8). It is useful to define the consideration set as those options $B=\{a \in A \mid p(a)>0\}$ that are chosen with positive probability. Proposition 1 gives a sufficient and quite intuitive condition for some paths to be omitted from the consideration set. The condition is not necessary: paths can be excluded from the consideration set even if they are not dominated by some other option. We will see examples of this in our numerical results below.

Proposition 1. (Matejka and McKay, 2015)Suppose that option a is dominated by option $d$ in the sense that $\forall \omega \in$ $\Omega: c(a \mid \omega) \geq c(d \mid \omega)$ with strict inequality for some $\omega$. Then $p(a)=0$.

Caplin et al. (2016) derived a necessary and sufficient condition for deciding which options belong to the consideration set and provided an algorithm for the solution.

Proposition 2. (Caplin et al., 2016) The information strategy $P(A \mid \Omega)$ is optimal if and only if for all $a \in A$,

$$
\sum_{\omega \in \Omega} \frac{\exp (-c(a \mid \omega) / \lambda) p(\omega)}{\sum_{a^{\prime}} \exp \left(-c\left(a^{\prime} \mid \omega\right) / \lambda\right) p\left(a^{\prime}\right)} \leq 1
$$

with equality if $p(a)>0$.

To compute $p(a), a \in A$, we begin with a guess for $p_{0}(a), a \in A$. The initial guess is iteratively updated until convergence with the next conditional probability vector computed from the previous according to

$$
P_{l+1}(a)=\sum_{\omega \in \Omega} \frac{\exp (-c(a \mid \omega) / \lambda) p(\omega)}{\sum_{a^{\prime}} \exp \left(-c\left(a^{\prime} \mid \omega\right) / \lambda\right) p\left(a^{\prime} \in A\right)} P_{l}(a) .
$$

The iteration of (10) will converge when the conditions in Proposition 2 are satisfied (with some chosen precision). In each iteration, the term in the bracket of (10) indicates whether the $P_{l}(a)$ needs to be raised or dropped. The options with $P_{l}(a)<\epsilon$ is set to be 0 . The solution generated by this algorithm is optimal if the initial consideration set with $P_{0}(a)>0$ is sufficiently large that it includes all possible choice options. In our study, as we can enumerate all the possible paths, the solution generated by the algorithm is guaranteed to be the optimal solution for the rational inattention problem.

Let $\hat{P}=P(A \mid \Omega)$ be the optimally chosen information strategy. Following the proof in Caplin et al. (2016) we substitute (8) into (4) to obtain

$$
\Lambda(\hat{P})=-\lambda \sum_{\omega \in \Omega} p(\omega) \log \left[\sum_{a \in \mathcal{A}} p(a) \exp (-c(a \mid \omega) / \lambda)\right] .
$$

This expression generalizes the logsum from the classic logit model.

Using the envelope theorem on the payoff (3), with the optimally chosen information strategy, we immediately obtain the following proposition.

Proposition 3. The traveler's optimal total travel cost increases with the unit information cost, i.e.

$$
\frac{\partial \Lambda(\hat{P})}{\partial \lambda}>0 \text {. }
$$




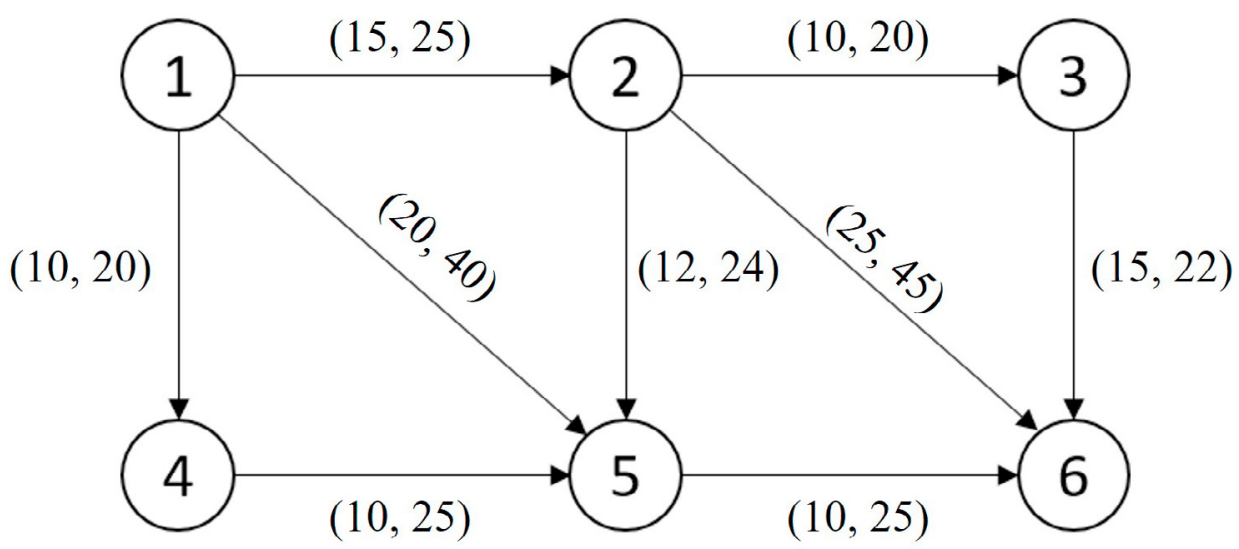

Fig. 5. A simple network for illustration

Proposition 3 is consistent with the intuition that information is helpful to reduce the total travel cost from the perspective of a single traveler. If the unit information cost is increased, then the traveler will acquire less information and be less able to adjust his choice of path to take varying travel times into account. Therefore his expected total travel cost increases.

\subsection{Numerical study}

In this section, we present the result of a numerical study to illustrate the behavior of a single rationally inattentive traveler facing a network with uncertain link travel times. The example network is shown in Fig. 5. There are 6 nodes and 9 links in the example network. The origin is node 1 and the destination is node 6 . For simplicity, we assume each link has two states, congested and non-congested. The two states occur with equal probability and the links are independent. There are then $2^{9}$ possible states. The traveler chooses between the 5 feasible paths from the origin to the destination, as listed in Table 2. There is no dominance relationship between the paths. Each path is the shortest path in some state.

Table 1. Enumeration of paths

\begin{tabular}{llll}
\hline Path ID & Node sequence & Possible values & Expected cost \\
\hline 1 & $1-2-3-6$ & $40,47,50(2), 57(2), 60,67$ & 53.5 \\
2 & $1-2-5-6$ & $37,47,49,52,59,62,64,74$ & 55.5 \\
3 & $1-5-6$ & $30,45,50,65$ & 47.5 \\
4 & $1-4-5-6$ & $30,40,45(2), 55(2), 60,70$ & 50 \\
5 & $1-2-6$ & $40,50,60,70$ & 55 \\
\hline
\end{tabular}

The optimal choice probabilities for the rationally inattentive traveler depend on the unit information cost $\lambda$. To illustrate its impact, Fig.6 depicts the optimal behavior of the traveler with different values of $\lambda$. For each path, we calculate three probabilities. $P(S P=i)$ denotes the unconditional probability of the path to be the shortest path; $P(A=i)$ is the unconditional probability of choosing path $i$ before receiving the signal; $P(S P=i \mid A=i)$ is the posterior probability of a path to be the shortest path (SP) conditioned on it being chosen. Among these three, $P(S P=i)$ is determined by the distribution of $\Omega$, which can be viewed as a characteristic of each path, thus is not affected by the value of $\lambda$.

First, we compare the size of the consideration set $B=\{a \in A \mid p(a)>0\}$ for different $\lambda$. When information is cheap, $\lambda=1$, the consideration set comprises all alternatives, $|B|=5$, which means that each path is chosen with positive 

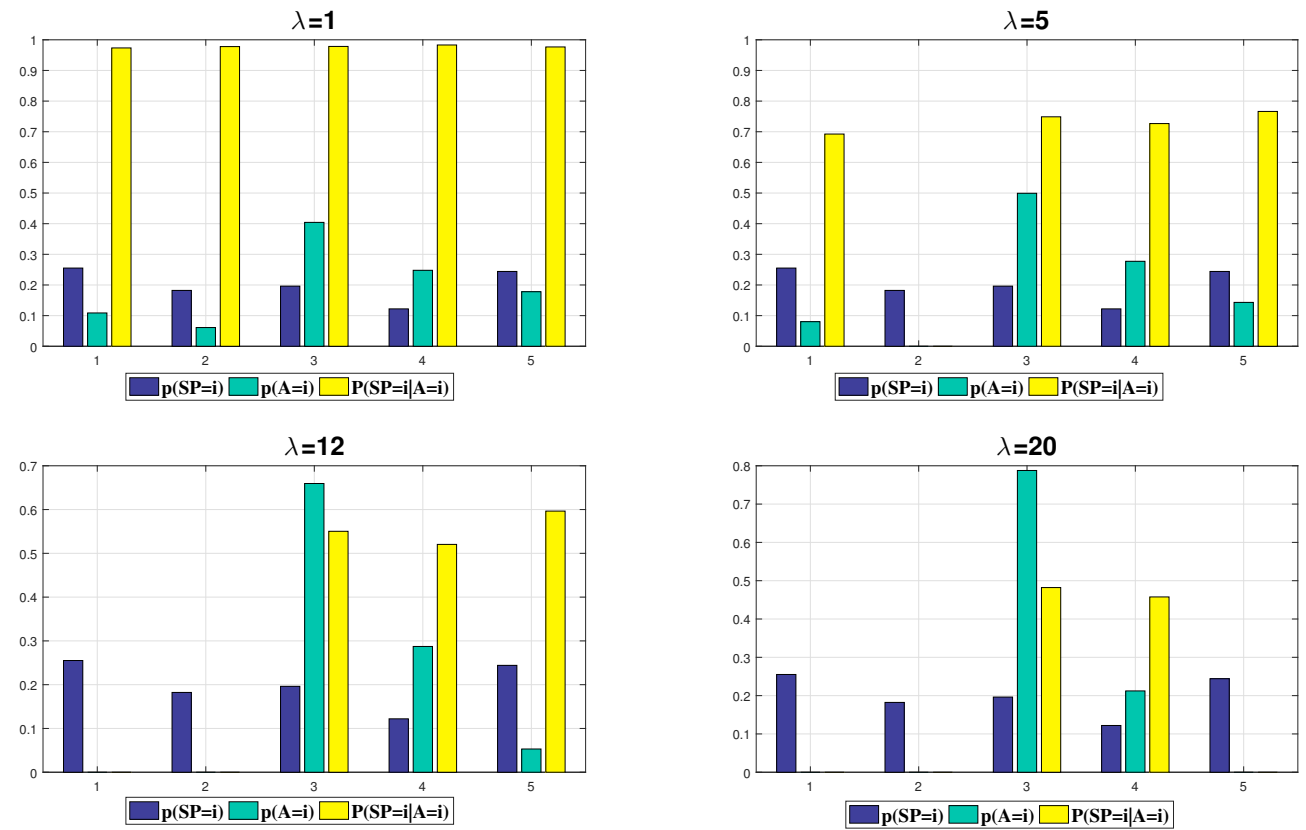

Fig. 6. Probability distributions for each path

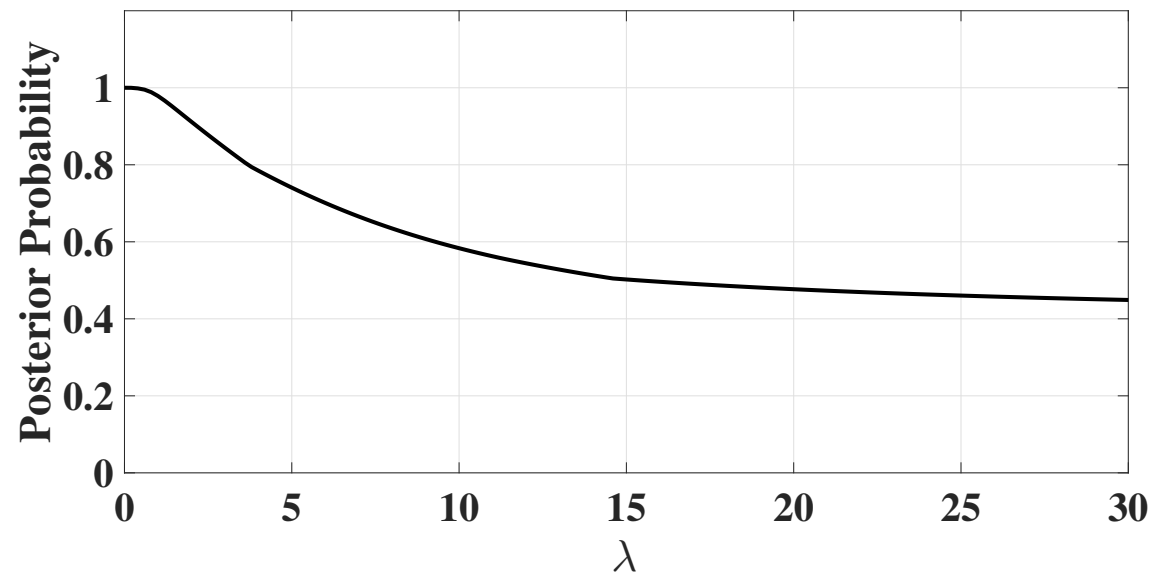

Fig. 7. Posterior probability of choosing the shortest path

probability. At $\lambda=5$, path 2 , the path with the smallest unconditional probability at $\lambda=1$, is dropped from the consideration set, which now has size $|B|=4$. The unconditional choice probability of path 1 is also very small. As $\lambda$ increases to 12, path 1 is dropped from the consideration set. At $\lambda=20$, only paths 3 and 4 remain in the consideration set. In the extreme case where $\lambda=\infty$, travelers will not acquire any information and they choose path 3 every day, as that path has the smallest expected cost in the absence of information. Altogether, as we would expect, the size of the consideration set decreases with the unit information cost. Intuitively, as information becomes more expensive, travelers will limit their attention to a smaller number of paths, paying more attention to paths that have a higher probability of yielding the least cost. 


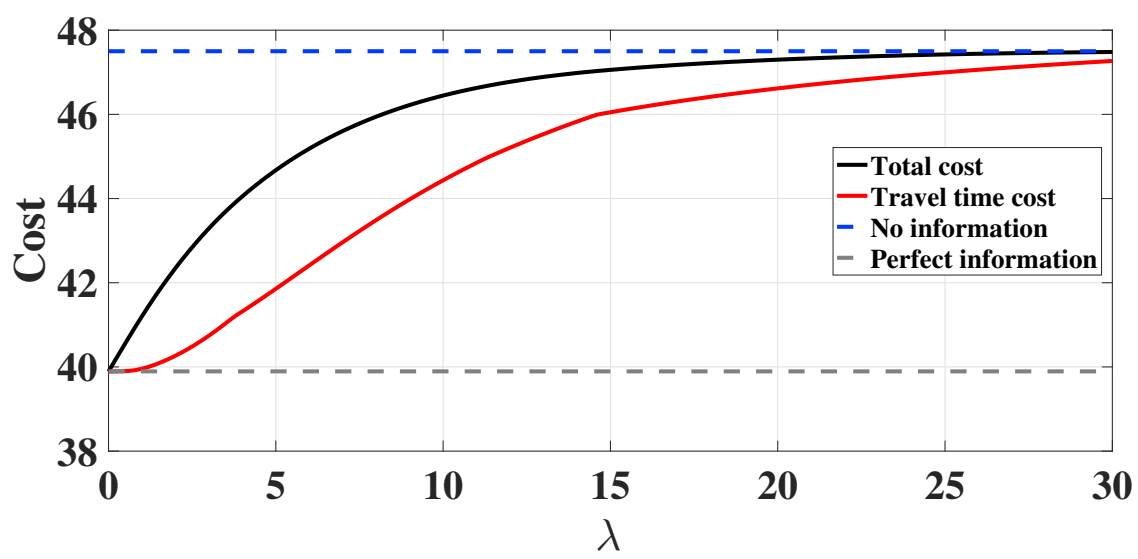

Fig. 8. Travel cost for different $\lambda$

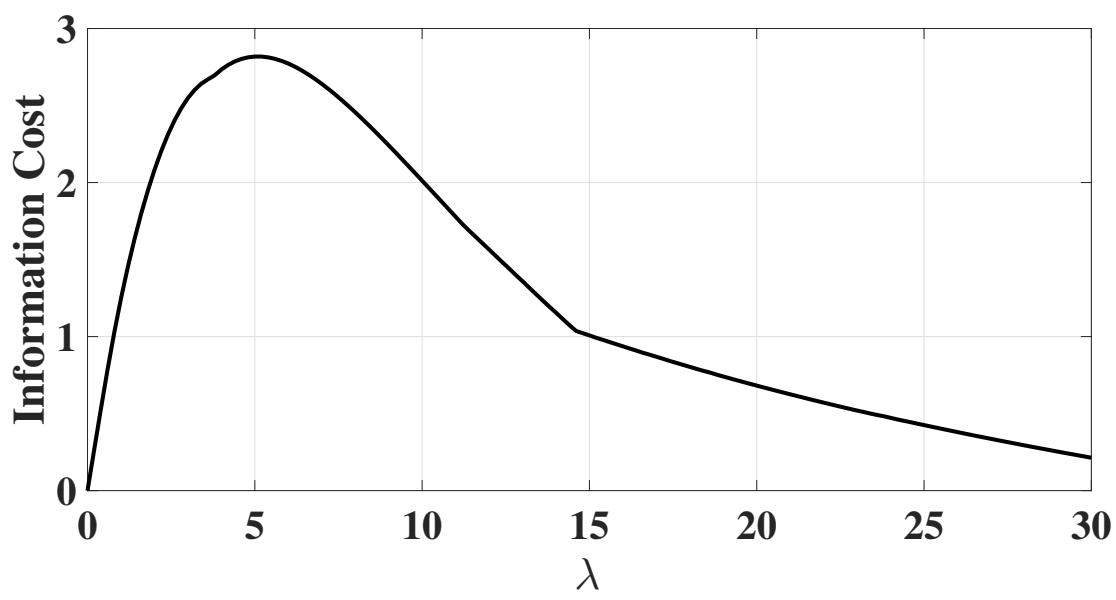

Fig. 9. Information cost for different $\lambda$

Another observation is that the posterior probability of choosing a better path decreases with information cost. When $\lambda=1$, the posterior probability of each alternative to be the shortest path is almost 1, i.e. the traveler is almost always able to choose the shortest path. When $\lambda=20$, there are only two options in the consideration set, and the posterior probability for each chosen path to be the shortest path is less than 0.5 .

Fig.7 shows the overall probability of choosing the shortest path. When $\lambda=0$, the information is free and the travelers get perfect information. They can choose the shortest path in each state with $100 \%$ probability. In Fig.7, the posterior probability decreases sharply for $\lambda<10$ and then decreases more slowly. This means that the marginal effect of the unit information cost is large when then information is cheap. Fig. 8 exhibits this characteristic as well. We can see the total cost and the travel time cost increases fast at small values of $\lambda$ and then the slope becomes small. Fosgerau and Jiang (2019) find similar results. It may be due to the sensitivity of the amount of information to the information cost. When $\lambda$ is relatively small, travelers acquire rich information and are thus very sensitive to states. In each state, the conditional probability $p(a \mid \omega)$ of the non-optimal paths are close to 0 . Due to the logarithm in the expression in the Shannon entropy. it is sensitive near 0. Therefore, the amount of information acquired decreases sharply in small $\lambda$, which leads to its large marginal effect on both the travel costs and on the overall posterior probability of choosing the shortest path. The blue and red lines in Fig. 8 represent the travel costs without information and with perfect information respectively, which are the boundary values of travel costs for the rational inattention model. In this example, the information may reduce up to $20 \%$ of travel cost compared to no-information scenario. 
Fig.9 shows the optimal information cost at different values of $\lambda$. The optimal information cost is zero when information is free or when information is so expensive that no information is obtained. It is positive in between, which makes the optimal information cost a non-monotonic function of $\lambda$. For small $\lambda$, a change in $\lambda$ dominates the change in the quantity of information acquired. The opposite happens for large $\lambda$. Comparing the total travel cost, travel time cost and information cost in Fig.8 and Fig.9, we find that the information cost only accounts for a small percentage of the total travel cost. However, it affects the route choice behavior and the travel cost to a great extent.

Now consider for a moment that there exists a path 6 that is a duplicate of path 3 . The travel time of the two paths are exactly the same in all states, i.e. $p(3 \mid \omega)=p(6 \mid \omega), \forall \omega \in \Omega$. We can think, perhaps, that we account separately for two parallel lanes on path 3 . As $p(a \mid \omega)$ has a logit-like form, one may worry that the model will produce unrealistic results resulting from the IIA property of logit model. However, Matejka and McKay (2015) proved that the duplicate actions are treated as a single action. Our numerical studies verified that adding a duplicate path does not affect the probability distributions of other paths. Compared to the case without the duplicate path, we have $p^{\prime}(3)+p^{\prime}(6)=p(3)$, where $p^{\prime}(a)$ is the unconditional probability of path $a$ after adding the duplicate path. Since the traveler is now indifferent between path 3 and 6 , there are infinite solutions to (3), as long as the sum of $p(3)$ and $p(6)$ is fixed. This result is a general property of the rational inattention logit model (Matejka and McKay, 2015) and is an important reason to prefer the rational inattention logit model over the plain logit model.

\section{User Equilibrium for rationally inattentive travelers}

Having considered the case of a single rationally inattentive traveler, we now proceed to consider the more complicated case of user equilibrium with rationally inattentive travelers.

\subsection{Problem formulation for a generalized network with heterogeneous travelers}

In Section 3, the link travel time was assumed to be independent of the route choice of the traveler. In this section, we generalize the model to a group of travelers in a stochastic network. The link travel time depends on the traffic flow and the realized capacity of the network. The randomness comes from the capacity of the network. The set of states $\Omega$ now includes all possible realization of capacity on each link in the network. The total demand from origin $O$ to destination $D$ is $N$. Each day, travelers choose between the paths, denoted as $a \in A$, where $A$ is the set of paths. Travelers are treated as a continuum, with every zero mass traveler making choices taking the choices of everybody else as given. The state-dependent travel time on every link is the prior knowledge of travelers. As the travelers do not consider the choices of others when making the choice, only in equilibrium their prior knowledge is consistent with the actual travel time they experienced.

For the sake of comparison, we introduce two extreme information scenarios in addition to the general rationally inattentive scenario. In the first extreme scenario, travelers have zero information cost. In this case, travelers obtain perfect information and the problem degenerates day by day to the deterministic model. On each day, the paths with positive flows will have the same cost, which is not larger than the cost on the unchosen paths. In the second extreme scenario, information is infinitely expensive and travelers obtain no information. They choose among paths based only on their prior knowledge of $p(\Omega)$. In equilibrium, the expected cost is constant across the chosen paths and not larger than the cost of the unchosen paths. However, since the realized capacities vary every day, the actual cost on each day may be different on the two paths.

The general case is in between these extremes. Travelers have positive and finite information cost. As there is a continuum of travelers, each individual traveler has no impact on the equilibrium and therefore has the same objective function (4) as the single individual traveler.

Assume there are $m$ types of travelers with heterogeneous unit information $\cos t\left\{\lambda_{1}, \lambda_{2}, \ldots, \lambda_{m}\right\}$. The mass of type $k$ travelers is $N_{k}$. Travelers with smaller $\lambda_{k}$ have cheaper access to information. The information cost has impact on the behavior of travelers and in return changes the impact of information itself. The rationally inattentive user equilibrium (RIUE) is then formulated through the following equations.

$$
p_{k}(a \mid \omega)=\frac{\exp \left(-c(a \mid \omega) / \lambda_{k}\right) p_{k}(a)}{\sum_{a^{\prime}} \exp \left(-c\left(a^{\prime} \mid \omega\right) / \lambda_{k}\right) p_{k}\left(a^{\prime}\right)}
$$




$$
\begin{aligned}
& p_{k}(a)=\sum_{\omega \in \Omega} p_{k}(a \mid \omega) p(\omega) \\
& n(a \mid \omega)=\sum_{k} N_{k} p_{k}(a \mid \omega) \\
& n(i \mid \omega)=\sum_{a} n(a \mid \omega) \epsilon_{i}^{a} \\
& c(a \mid \omega)=\sum_{i} c(i \mid \omega) \epsilon_{i}^{a}
\end{aligned}
$$

We note the following properties of RIUE:

(i) Travellers with the same $\lambda_{k}$ has the same generalized travel cost.

(ii) The state-dependent link flow is deterministic. Each day travelers get information about the traffic state according to their information strategies. Every state has a specific equilibrium so the link flow pattern changes every day depending on the realized state.

(iii) Travellers' prior knowledge of the state-dependent travel time are the same as the equilibrium travel time. Before the equilibrium is achieved, the state-dependent travel time, which is treated as the prior knowledge of travelers, is not the same as the true travel time the travellers experience. Because the each traveler makes the choice without considering the route choice of others. Only in equilibrium, the prior knowledge of travel time is unbiased.

The case with homogeneous travellers is a special case of the heterogeneous travellers if we set $k=1$. If the travellers have same unit information cost, in equilibrium their total travel costs are the same. Generally, $p_{k}(a \mid \omega)$ distinguishes between different values of $\lambda$.

Finally, we define

$$
c(i \mid \omega)=t_{i}^{0}\left(1+\beta\left(\frac{n(i \mid \omega)}{s(i \mid \omega)}\right)^{\gamma}\right)
$$

where $t_{i}^{0}$ is the free flow travel time of link $i$ and $n_{i}$ is the traffic flow on link $i$. s $\left.i \mid \omega\right)$ is the realized capacity of path $i$ under state $\omega$, which is the source of randomness.

We establish that the equations defining the RIUE always have a solution.

Proposition 4. The equilibrium defined by equations (12)-(17) exists.

Proof. Equations (14) - (17) define the cost matrix $c(A \mid \Omega)$ as a continuous function of the conditional choice probability matrixes $p_{k}(A \mid \Omega)$. Similarly, (13) defines the unconditional probabilities as a continuous function of the conditional choice probability matrixes. Then (12) is a continuous fixed point equation on the convex and compact set of conditional choice probability matrixes. By the Brouwer fixed-point theorem, the solution exists.

We are not able to guarantee that equilibrium is unique. This is due to mathematical complexity and it may be that there are general conditions under which equilibrium is necessarily unique. The uniqueness of the problem is left for future study.

Proposition 5. The generalized travel cost of travelers with cheaper unit information cost is smaller than those with larger unit information cost, i.e. if $\lambda_{k}<\lambda_{j}$, then $\Lambda_{k}<\Lambda_{j}$.

Proof. In equilibrium, the state-specific cost on each path is the same for all traveler types. Travelers with different $\lambda$ have distinct information strategy and generalized cost. Thus, Proposition 3 directly leads to the statement in Proposition 5.

The user equilibrium in transportation network has been studied for many decades. There are many pioneer research on deterministic user equilibrium (DUE) (Daskin, 1985). Since the DUE cannot capture the uncertainty and randomness in reality, researchers have developed the stochastic user equilibrium (SUE) models which consider the perception error of the travel cost (e.g., Bell, 1995; Yang, 1997). Unlike the general path-based SUE models Fosgerau et al. (2013) and Mai et al. (2015) proposed the link-based recursive logit (RL) and the nested recursive logit 
(NRL) model. In SUE, the actual travel time is still deterministic. The reliability-based stochastic user equilibrium (RSUE) deals with the network with uncertain travel time (e.g., Shao et al., 2006; Lam et al., 2008; Lo et al., 2006). Recently, researchers have proposed the bounded rational user equilibrium (BRUE) (Di et al., 2013), assuming the travel cost differences of the paths with positive flow are within an indifference band of travelers. Actually, RIUE is a kind of BRUE model which takes advantages of imperfect information. To better understand the proposed RIUE, the comparison of the mentioned models are listed in Table 2.

Table 2. Comparison of UE models

\begin{tabular}{llll}
\hline Model & Stochastic network & Information & Perceived error \\
\hline DUE & No & No & Yes \\
RSUE & No & Yes/No & No \\
RL & No & No & Yes \\
NRL & No & No & Yes \\
BRUE & No & No & No \\
RIUE & Yes & Yes & No \\
\hline
\end{tabular}

The social welfare is measured by the total travel cost of all the travelers, including the information cost for the rationally inattentive travelers. The information cost for the uninformed travelers is zero.

Having a model that explicitly includes information allows us to answer questions related to the provision of information. Intuition suggests that making information more easily accessible (and hence cheaper to acquire) will be welfare improving. However, Lindsey et al. (2014) have shown through counter-examples that information is not always welfare-improving since congestion externalities may increase. It is then of interest to see if this conclusion remains in the rational inattention model. The conclusion may change since the rational inattention model endogenizes the acquisition of information and the cost of information is part of the welfare measure. Following the earlier literature, we investigate the issue by means of stylized examples.

\subsection{Solution algorithm}

The solution algorithm is as follows. We initialize $c_{0}(a \mid \omega)$ of each path as the free flow travel time. According to (13) - (16), the $c(a \mid \omega)$ is updated after each iteration until $c(a \mid \omega)$ converges to the equilibrium travel cost. Correspondingly, the equilibrium path flow can be calculated. The solution algorithm is possibly interpreted as the learning behavior of travelers depicted in Fig.10. But the actual learning process of travelers will need more empirical evidences for calibration or validation. In (12)-(13), the state-specified path cost $c(a \mid \omega)$ and the probability distribution of states $p(\omega)$ are the prior knowledge of travelers. In the evolution, each traveler makes the route choice without considering the choices of others. In equilibrium, the travelers will find their prior knowledge of travel time consistent with the experienced travel time distribution. Otherwise, they keep updating their knowledge of $c(a \mid \omega)$. In equilibrium, each state of capacity corresponds to a distinct traffic flow.

\subsection{Numerical example}

The numerical example employs a two-link network, similar to those used by Lindsey et al. (2014) and Emmerink et al. (1998) for studying the impact of information. Our example differs from the previous in the following two ways: (1) In the rational inattention model, the information strategy is endogenously chosen by travelers; (2) We allow traveler heterogeneity.

\subsubsection{A freeway and an arterial road}

Consider a network with two links, each corresponding to a path as shown in 11. Path 1 is a safe path and path 2 is a risky path with random capacity. More specifically, path 1 is the freeway with deterministic travel time $t_{f}$ which does not change with traffic flow. Path 2 is an arterial road with BPR cost function (17). The path 2 has stochastic 
The free flow travel time and capacity distribution is known to travelers as prior knowledge

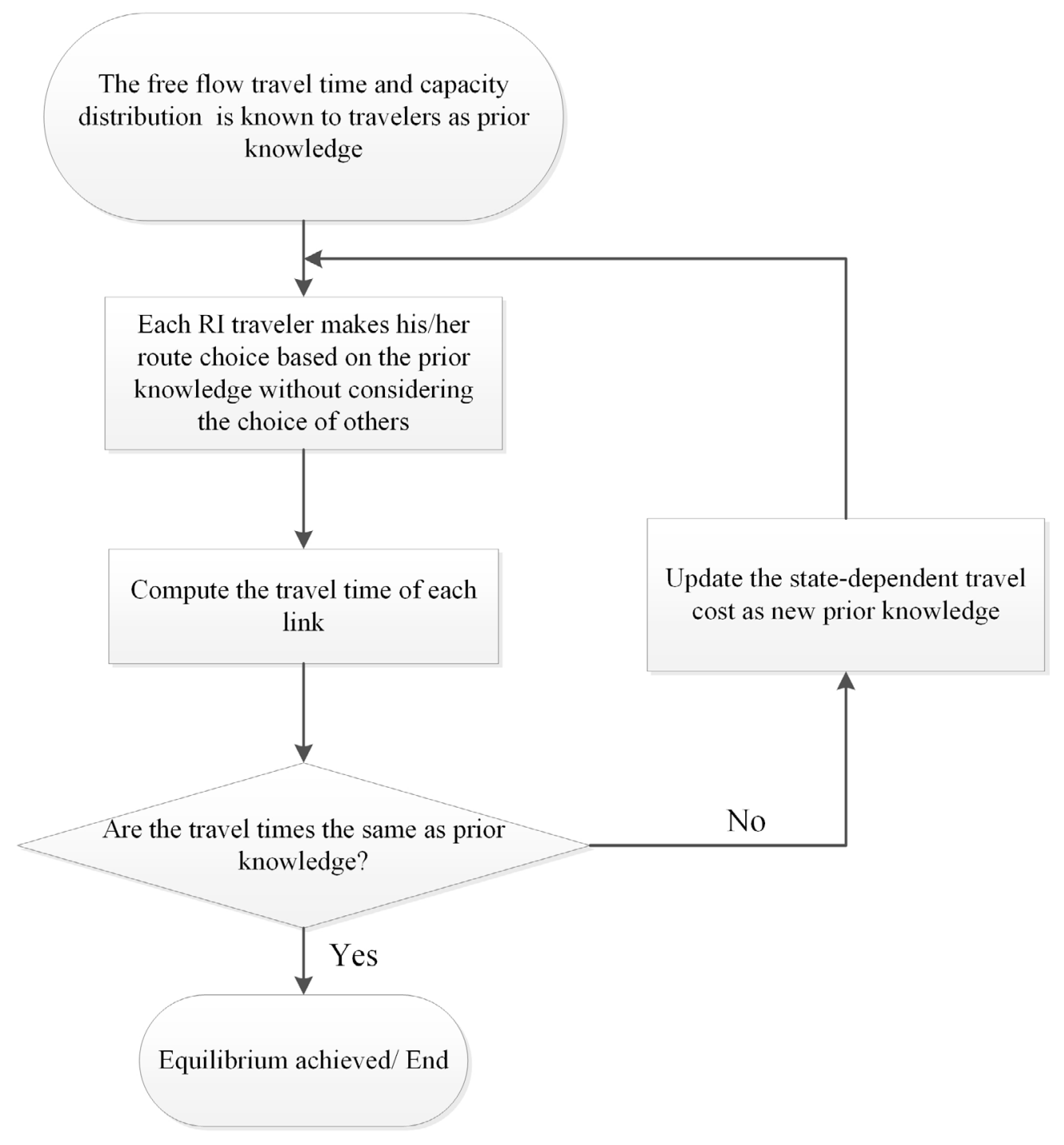

Fig. 10. The updating process of travelers

capacity $S_{2}$ and free flow travel time $t_{0}$. The example is constructed such that one path does not dominate the other. The parameters are listed in Table 3. The distribution of $S_{2}$ is assumed to be uniform. The possible values of $S_{2}$ define the states. The probability distribution of $\Omega, p(\Omega)$, is known to travelers. Emmerink et al. (1998) investigated the impact of imperfect information on a one-link network with elastic demand. They adopted a linear demand and cost functions. In this example, the freeway is introduced to investigate the elastic demand on the arterial road without specific assumptions about the demand function. The total demand is $N$. There are two types of travelers with imperfect information and without information respectively. The proportion of uninformed travelers is $\delta$.

Fig. 12 shows the conditional probability of choosing the risky path in the different states. The uninformed travelers equalize the expected travel cost of the two paths and the probability of choosing each path does not vary with the state. In contrast, the rationally inattentive travelers acquire information that leads them to increase their probability of choosing the risky path in states when the capacity is high.

The right panel of Fig.12 shows the travel time on the risky path in the different states. We observe that the travel time on the risky path decreases with capacity even though the demand increases. The uninformed travelers take into account an expected travel cost on the risky path that in equilibrium is equal to the cost on the safe path, which is 15 . 
Table 3. Parameters used in the numerical example

\begin{tabular}{lll}
\hline Variables & Symbol & Value \\
\hline Travel time of path 1 & $t_{f}$ & 15 \\
Free-flow travel time of path 2 & $t_{0}$ & 5 \\
Maximum capacity of path 2 & $S_{2}^{\max }$ & 40 \\
Travel demand & $N$ & 150 \\
Degradable parameter & $\theta$ & 0.4 \\
Unit information cost & $\lambda$ & 10 \\
Percentage of uninformed travelers & $\delta$ & 0.5 \\
Parameters for BPR function & $\beta$ & 0.15 \\
& $\gamma$ & 4
\end{tabular}

Path 1

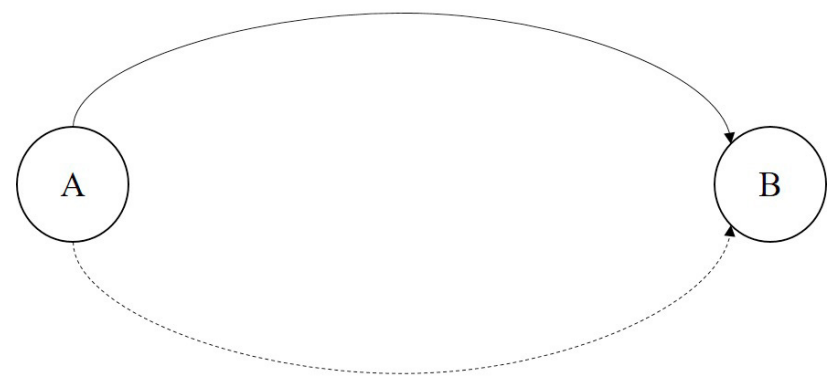

Path 2

Fig. 11. Degradable network

We have calculated the expected travel cost of rationally inattentive travelers and find that their travel time cost is 14.57 and their information cost is 0.21 . We can see that the information cost only accounts for a small part of the total generalized cost, but it leads to significant changes in the traffic behavior. We can link this result to reality that usually it does not take us much effort to search for some rough information about the traffic states. However, the information we get has large impact on our behavior.

Fig.13 shows the unconditional probability of choosing the risky path for different values of $\lambda$ (left panel) and $\delta$ (right panel), where $\delta$ is the percentage of uninformed travelers. In the left panel, $\delta$ is kept constant and the unit information cost is varied. As $\lambda$ increases, the unconditional share of rationally inattentive travelers on the risky path increases a little while the share of uninformed travelers decreases due to equilibrium. The overall usage of the risky path decreases with $\lambda$. The story can be told as this: when information is more expensive, rationally inattentive travelers are less able to avoid the risky path on bad days, which worsens the path condition. Since the uninformed travelers cannot get information, the unconditional probability of uninformed travelers are more sensitive to the bad condition than the rational inattentive travelers. Therefore, the uninformed travelers move the other way to avoid the high cost on bad days. Due to equilibrium, the rational inattentive travelers use the risky path more.

In the one-link network of Emmerink et al. (1998), both the uninformed travelers and informed travelers use the path more due to information. Our results is slightly different from theirs, possibly resulting from assumptions of demand and supply functions. In their one-link network, there is no equilibrium considered. Instead, they used a linear demand function. But one thing in common is that the information increases the usage of the variable path. In the right panel, it is interesting to observe that the unconditional probability of choosing path 2 increases with the percentage of uninformed travelers $\delta$ for both uninformed and rational inattentive travelers while the total usage of path 2 decreases with $\delta$. This may seem contradictory but note that $P_{2}=P_{2}^{u n} * \delta+P_{2}^{R I} *(1-\delta)$, where $P_{2}^{u n}$ and $P_{2}^{R I}$ is the probability of choosing the risky path for uninformed and rational inattentive travelers, respectively. As $\delta$ increases, some travelers change from $P_{2}^{R I}$ to $P_{2}^{u n}$. Although $P_{2}^{u n}$ is increasing, it is smaller than $P_{2}^{R I}$, resulting in the decrease in 

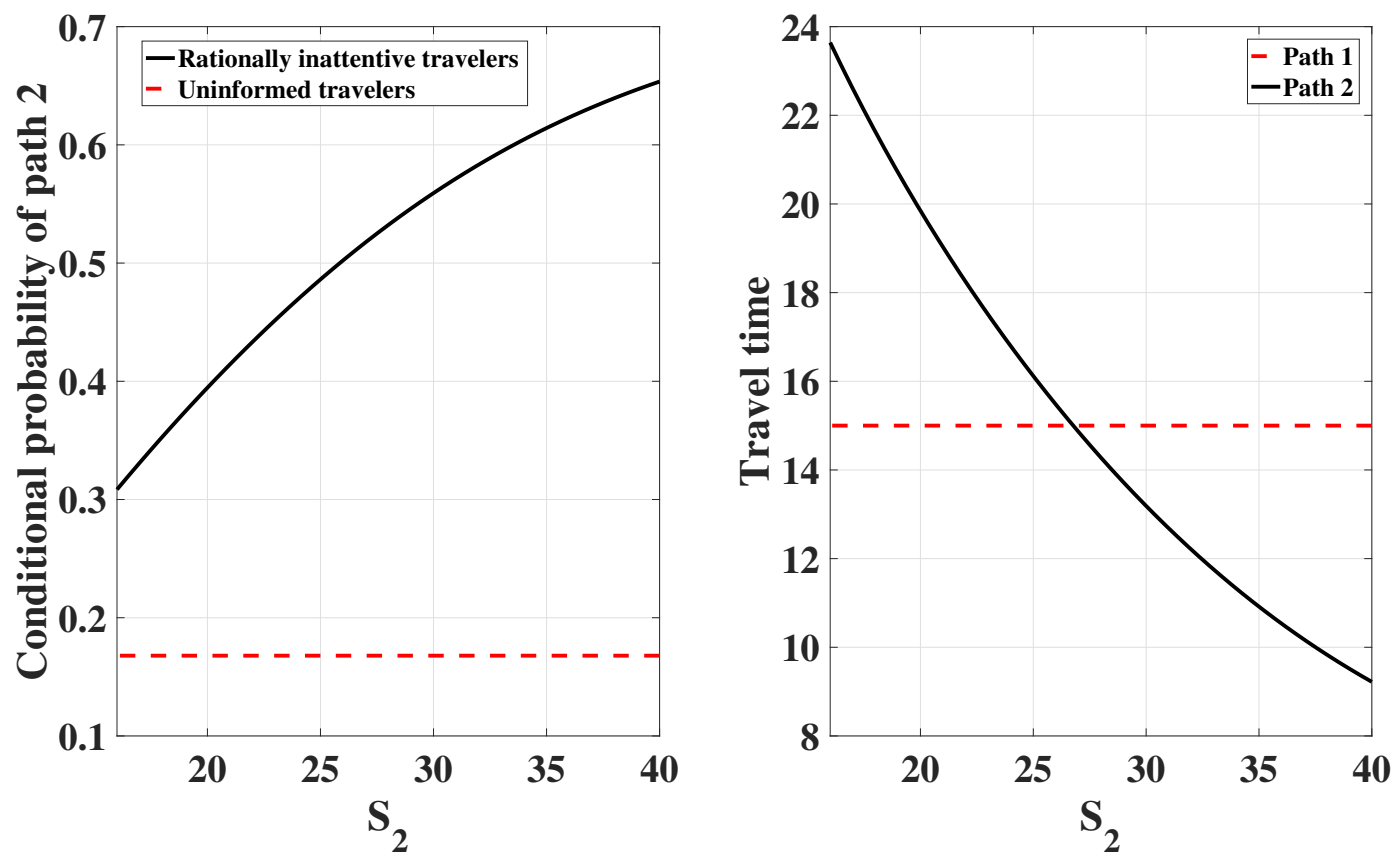

Fig. 12. Conditional probability and travel cost under different states
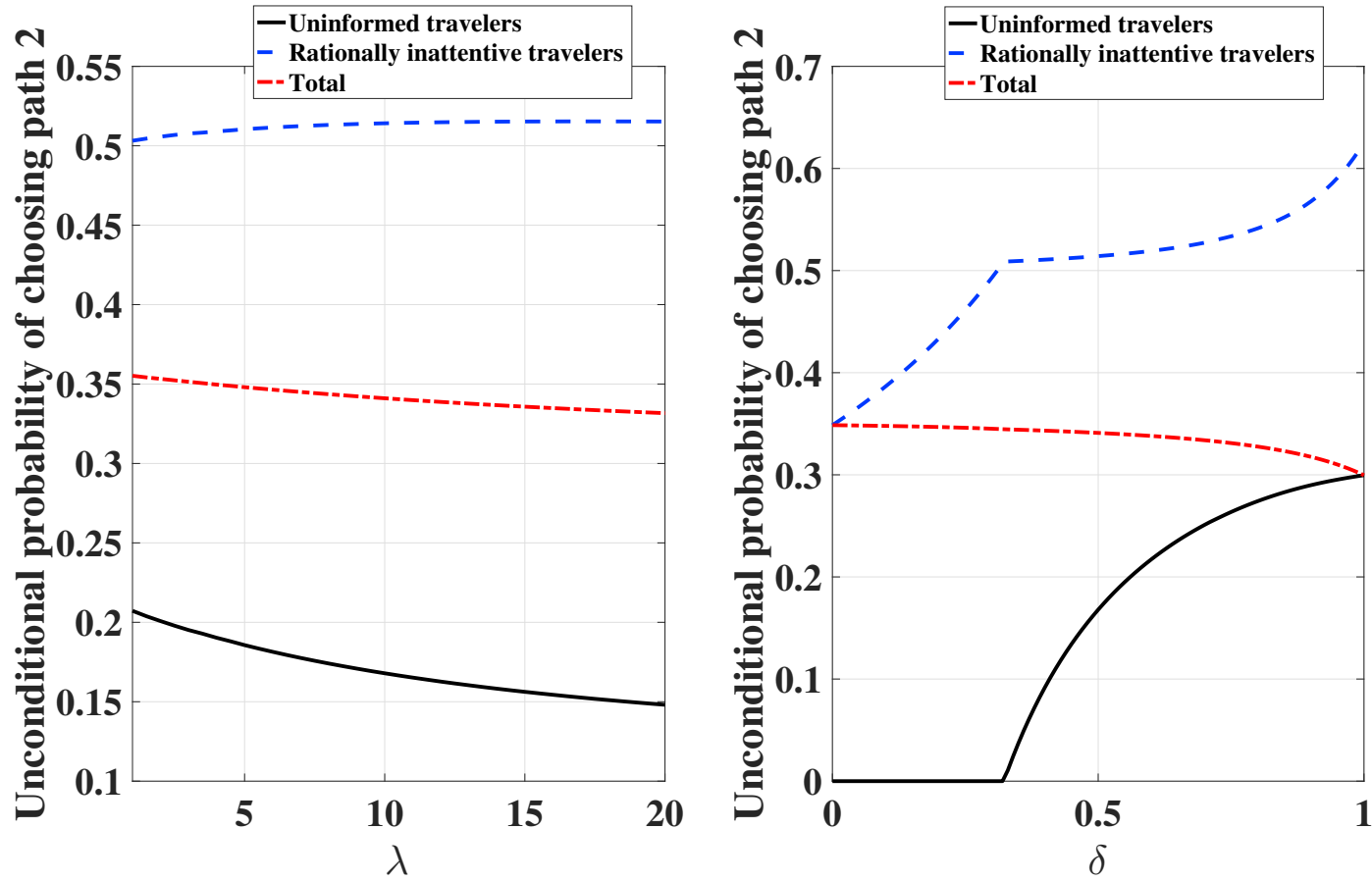

Fig. 13. Flow distribution 

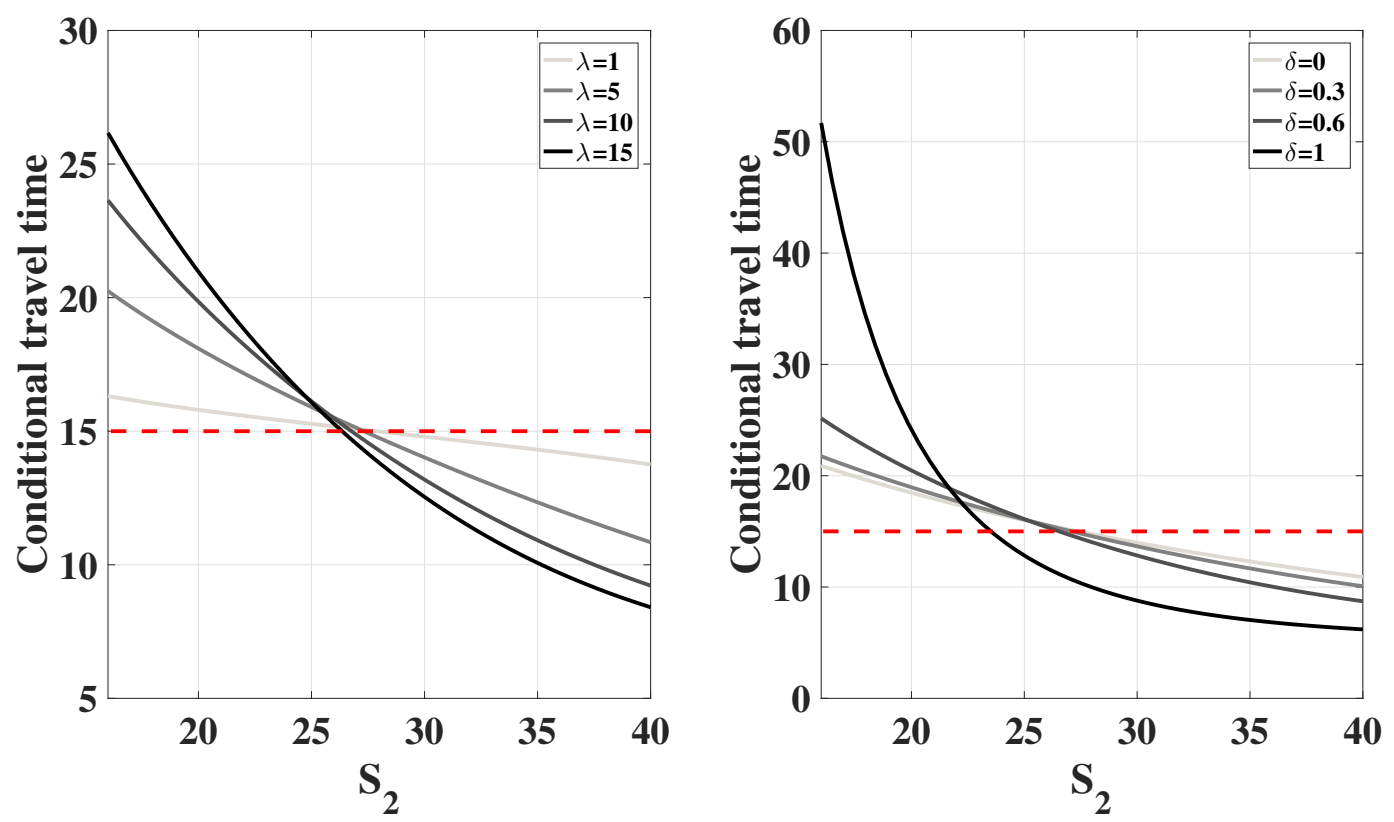

Fig. 14. Travel time of under different scenarios

the overall $P_{2}$. The figure illustrates that as the number of uninformed travelers decreases, usage of the risky path also decreases. This is because that travelers are less able to respond to variable traffic conditions.

If travelers could obtain perfect information, then, in equilibrium and in each state, the two paths would have equal cost. In the rational inattention model, as travelers have limited information, they cannot react to the capacity changes as well as they would have been able to with perfect information, so the travel time of the two paths are different when equilibrium is achieved. The lines in Fig.14 represents different values of $\lambda$ and $\delta$. The red line represents the static travel time on path 1 and the other represents the variable travel time on path 2 . As $\lambda$ decreases, the information is cheaper and the travelers get more information, the travel time of the two paths are closer under each states. As $\delta$ increases, the uninformed travelers increases and the cost difference of the two paths are much larger than that with smaller $\delta$. In summary, information reduces the cost disequilibrium of the two paths, especially when in extreme cases where the capacity of path 2 is very low or high.

Following Proposition 5, the generalized cost of rationally inattentive (RI) travelers should always be smaller than the uninformed travelers. As the expected travel cost of uninformed travelers equals to $t_{f}$, they are not sensitive to $\delta$ and $\lambda$. Therefore, the information reduces the travel time of rationally inattentive travelers while keping the expected travel cost of the uninformed travelers unchanged as $t_{f}$. Therefore, we can conclude that in this network, information is always welfare-improving relative to the case without information. However, the welfare improving effect is not monotonic with the amount of acquired information.

To examine the impact of imperfect information, Fig. 15 depicts the generalized cost for the rationally inattentive (RI) travelers, scaled travel time cost of all travelers, scaled information cost and scaled total cost including the travel time cost and information cost. The following formula is used for scaling:

$$
v^{\prime}=\frac{v-v_{\min }}{v_{\max }-v_{\min }}
$$

where $v^{\prime}$ is the scaled value of $v \cdot v_{\min }$ and $v_{\max }$ are the minimum and maximum of the original value.

In Fig. 15, the upper left panel depicts the generalized total cost for RI travelers. As mentioned above, the cost of uninformed travelers is $t_{f}$, which is 15 in this case. When the percentage of the uninformed travelers is not large, the generalized cost of RI travelers is not very sensitive to $\delta$ and $\lambda$. Only when the rationally inattentive travelers is the minority, they have an obvious advantage over the uninformed travelers. This may be interpreted as follows. When 

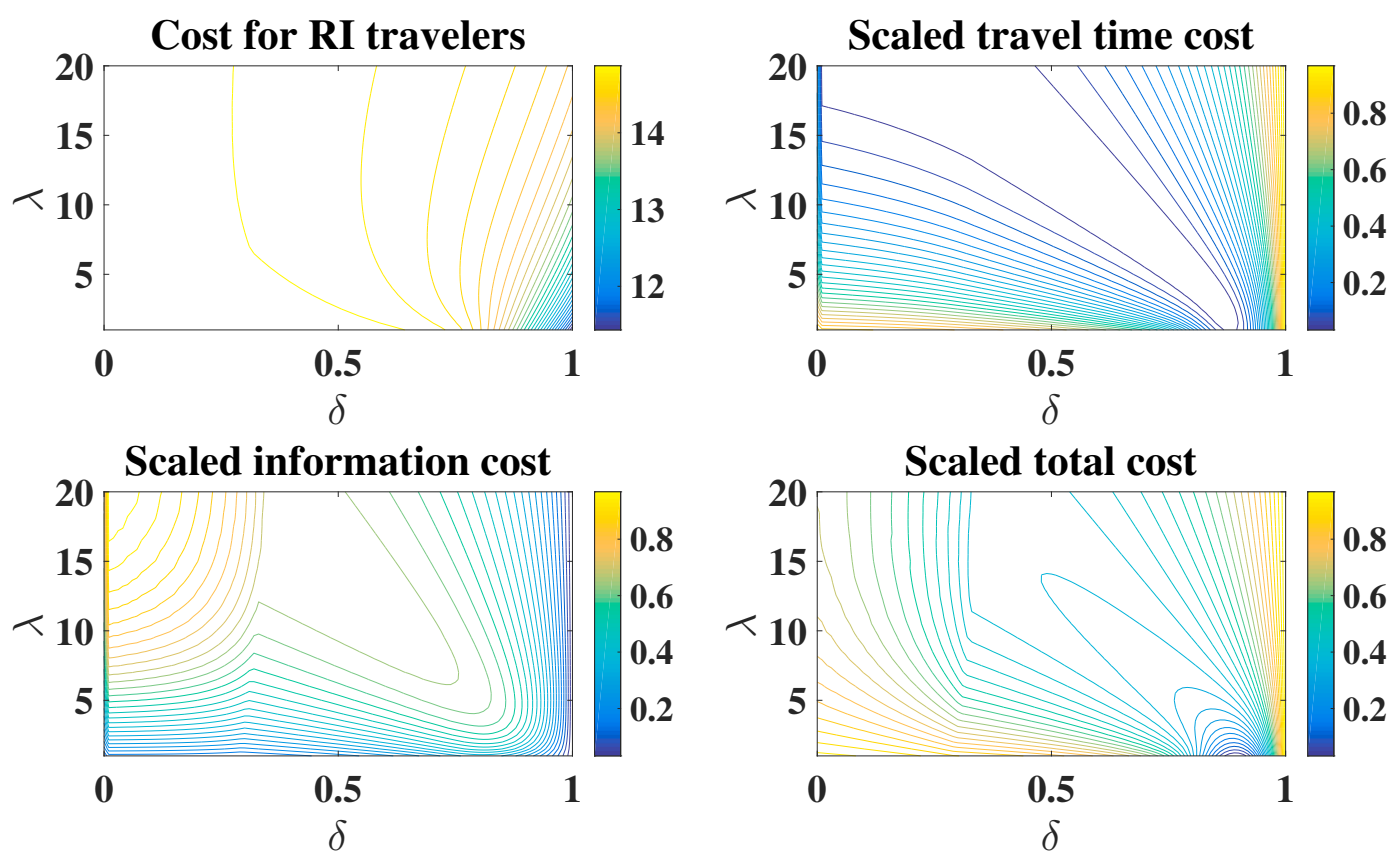

Fig. 15. Cost sensitivity for $\lambda$ and $\delta$

the information is cheap or the market share of RI travelers is large, the cost disequilibrium of the two paths are small, which lessens the difference in the cost between informed and uninformed travelers. When the majority of travelers are uninformed travelers, the travel time varies in different states, which allows the RI travelers to take advantage of the information so their benefit is more obvious.

In the upper right panel, it is interesting to observe that the total travel time in not monotonic with $\delta$ and $\lambda$. When the percentage of uninformed travelers is small, the total travel time decreases with $\lambda$, which means that less information is more preferable for the system. Although we proved that from the travelers' perspective, those endowed with information is always better than those without information. However, the information does not always reduce the total travel time of the whole system. Lindsey et al. (2014) explained that such a situation may occur because the shorter path with less free flow travel time is overused in equilibrium with perfect information. Similar arguments apply for the case with imperfect information. Only when the proportion of uninformed traveler is very large (bigger than 0.9), the total travel time decreases with $\lambda$, meaning that more information brings benefit to the system. The optimal travel time occurs when both $\delta$ and $\lambda$ are moderate, illustrating that the system behaves well when there are not too many informed travelers and the information cost is not too small. The lower panels are the total scaled information cost and the scaled total cost. Comparing the contour of travel time cost and total cost, most of the area with low cost overlaps. The scaled total cost exhibits similar characteristics as the scaled travel time cost. From the perspective of the planner, we may not be able to control the percentage of the uninformed travelers, but we may adjust the cost or accessibility of information, which is scaling parameter $\lambda$. From the perspective of a single traveler, obtaining information is always better if the exogenous conditions are unchanged. However, from the perspective of the whole network, too much information might not be preferable.

\subsubsection{Two stochastic paths}

This subsection further examines the impact of imperfect information on social welfare. Lindsey et al. (2014) stated that the perfect information is welfare-improving if the free flow travel time of the two links are always equal. Under this condition, the user equilibrium under perfect information coincides with system optimum (SO). As they did not consider heterogeneous travelers and imperfect information, we wonder whether the imperfect information is also beneficial for the network under different composition of travelers and information cost. Therefore we adopted similar 
parameters as in their study. In this two-link network, both the links are stochastic with two states: good and bad. The 'good state' corresponds to the case with large capacity and vice versa. Parameter values are set as follows: $N=120$, $t_{0}=5, S_{1 B}=20, S_{1 G}=35, S_{2 B}=25, S_{2 G}=40$. The capacity states of the links are independent of each other.

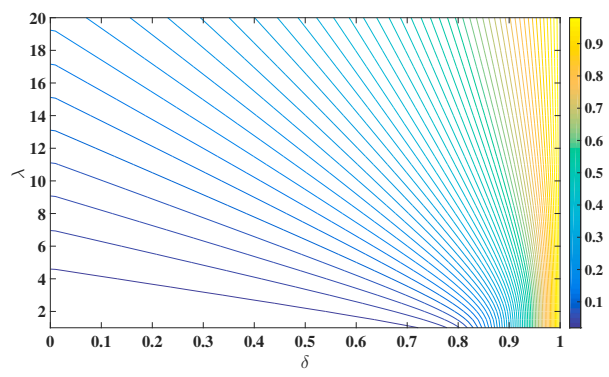

Fig. 16. Scaled total travel cost with independent path capacity

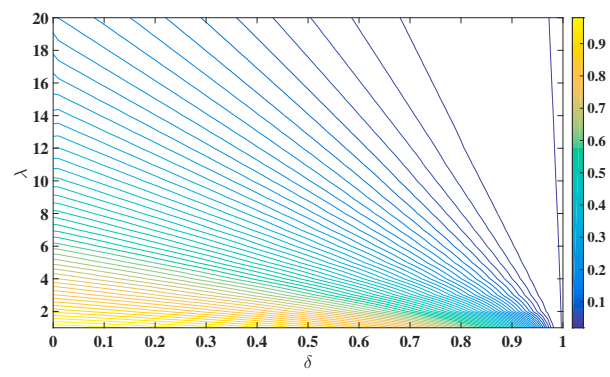

Fig. 17. Scaled total travel cost with correlated path capacity

As shown in Fig.16, the total travel cost increases monotonically with $\delta$ and $\lambda$. That means that in this network where perfect information is welfare-improving, imperfect information always decreases the total travel cost.

However, information is not always welfare-improving. Lindsey et al. (2014) found perfect information may be welfare-reducing when the free flow travel times of the two paths are not equal and the capacities are equiproportional (i.e., $S_{1 B} / S_{1 G}=S_{2 B} / S_{2 G}$ ). In this scenario, the perfect information may result in the overuse of the path with smaller free flow travel time. We changed the parameters to: $t_{1}=5, t_{2}=8 S_{1 B}=30, S_{1 G}=40, S_{2 B}=37.5, S_{2 G}=50$, where the states are perfectly correlated, meaning that if path 1 is in 'bad condition', the path 2 if in 'bad condition' as well. The scaled total travel time is depicted in Fig.17. We found that the imperfect knowledge is welfare-reducing compared to the case without information as well. Fig.16 and Fig.17 show the total travel cost with imperfect information in the network with two stochastic paths are intermediate between the case with perfect information and without information. Whether the imperfect information is welfare-improving or welfare-reducing depends on the impact of perfect information. In previous section where there is only one variable link with elastic demand, the information is always welfare-improving but imperfect information reduces the total travel cost more than perfect information. In this section, the impact of imperfect information is more moderate than the perfect information.

\section{Conclusion and discussion}

This paper has set up a rational inattention model for the path choice problem where the travel time is uncertain. The rational inattention model has the very attractive feature that it incorporates travelers' information strategy endogenously, taking into account that travelers choose how much information to possess before making the route choice. It relaxes the assumption in previous research where the information is exogenously given. Based on the framework of rational inattention model, the impact of imperfect information is extensively studied. In the path choice problem of a single traveler, we showed that the size of consideration set decreases with unit information cost. A path with large expected cost will not be considered, even it has a great chance of being the shortest path. The posterior probability to choose the best path and the total travel cost decrease with information cost, which illustrates that the information is helpful for travelers to react to the travel time uncertainty.

We further extended the rational inattention model to a network with heterogeneous travelers where the rationally inattentive user equilibrium (RIUE) is considered. The existence and uniqueness of the solution is discussed and some properties of RIUE are presented. In the numerical study, a two-link network with different settings are adopted to examine the impact of imperfect information, from both the perspective of travelers and the system total travel cost. Compared to the case without information, imperfect information is always beneficial in the case with a freeway and an arterial road. In the network with two variable links, the imperfect information may be welfare-improving or welfare-reducing, exhibiting the same characteristic with perfect information.

The are many interesting questions beyond this model. We might use the rational inattention model to determine consideration sets. As many researchers have investigated, some travelers exhibits route choice inertia in network equilibrium (Zhang and Yang, 2015). The consideration of information cost may partly explain the inertia as travelers 
do not want to waste their information acquisition on inferior routes with long travel times. The results of the rational inattention model can also be compared with the deterministic model and the logit model. In the deterministic model, each used route has exactly the same travel time. In the logit model, even a path with large travel time will still be used. The rational inattention model can produce more reasonable results than these two models. It allows cost differences among the used paths and it will eliminate some routes from the consideration set. Another issue is the empirical studies and calibration of unit information cost. Currently there are not many empirical studies employing the rational inattention model. It is meaningful to analyze the data of travel time, departure time, route choice to validate the rational inattention model for future prediction. Another issue for future research is the formulation of link-based rational inattention route choice models.

This study might be incorporated with other route choice studies concerning the risk attitudes of travellers where the travel cost not only consists the travel time but also the travel budget, schedule cost and etc. In summary, the rational inattention model is very attractive for studying the impact of information on travelling behavior.

\section{Acknowledgements}

The study is supported by General Research Fund \#615712 and \#616113 of the Research Grants Council of the HKSAR Government and the Hong Kong PhD Fellowship. Mogens Fosgerau is supported by an ERC Advanced Grant GEM (740369).

\section{References}

Arnott, R., De Palma, A., Lindsey, R., 1991. Does providing information to drivers reduce traffic congestion? Transportation Research Part A: General 25, 309-318.

Avineri, E., Prashker, J.N., 2006. The impact of travel time information on travelers learning under uncertainty. Transportation 33, 393-408.

Bell, M.G., 1995. Stochastic user equilibrium assignment in networks with queues. Transportation Research Part B: Methodological 29, 125-137.

Bifulco, G.N., Cantarella, G.E., Simonelli, F., Velonà, P., 2016. Advanced traveller information systems under recurrent traffic conditions: Network equilibrium and stability. Transportation Research Part B: Methodological 92, 73-87.

Caplin, A., Dean, M., Leahy, J., 2016. Rational Inattention, Optimal consideration sets and stochastic choice. Technical Report. Working paper.

Chen, A., Zhou, Z., Lam, W.H., 2011. Modeling stochastic perception error in the mean-excess traffic equilibrium model. Transportation Research Part B: Methodological 45, 1619-1640.

Daganzo, C.F., Sheffi, Y., 1977. On stochastic models of traffic assignment. Transportation science 11, 253-274.

Daskin, M.S., 1985. Urban transportation networks: Equilibrium analysis with mathematical programming methods.

Di, X., Liu, H.X., Pang, J.S., Ban, X.J., 2013. Boundedly rational user equilibria (brue): mathematical formulation and solution sets. ProcediaSocial and Behavioral Sciences 80, 231-248.

Emmerink, R.H., Verhoef, E.T., Nijkamp, P., Rietveld, P., 1998. Information policy in road transport with elastic demand: Some welfare economic considerations. European Economic Review 42, 71-95.

Ettema, D., Timmermans, H., 2006. Costs of travel time uncertainty and benefits of travel time information: conceptual model and numerical examples. Transportation Research Part C: Emerging Technologies 14, 335-350.

Fosgerau, M., Frejinger, E., Karlstrom, A., 2013. A link based network route choice model with unrestricted choice set. Transportation Research Part B: Methodological 56, 70-80.

Fosgerau, M., Jiang, G., 2019. Travel time variability and rational inattention. Transportation Research Part B: Methodological 120, 1-14.

Fosgerau, M., Melo, E., de Palma, A., Shum, M., 2017. Discrete choice and rational inattention: a general equivalence result. Working paper . Gao, S., 2012. Modeling strategic route choice and real-time information impacts in stochastic and time-dependent networks. IEEE Transactions on Intelligent Transportation Systems 13, 1298-1311.

Gao, S., Frejinger, E., Ben-Akiva, M., 2010. Adaptive route choices in risky traffic networks: A prospect theory approach. Transportation research part C: emerging technologies 18, 727-740.

Hogarth, R.M.R.M., 1987. Judgement and choice: The psychology of decision.

Lam, W.H., Shao, H., Sumalee, A., 2008. Modeling impacts of adverse weather conditions on a road network with uncertainties in demand and supply. Transportation research part B: methodological 42, 890-910.

Lindsey, R., Daniel, T., Gisches, E., Rapoport, A., 2014. Pre-trip information and route-choice decisions with stochastic travel conditions: Theory. Transportation Research Part B: Methodological 67, 187-207.

Liu, W., Li, X., Zhang, F., Yang, H., 2017. Interactive travel choices and traffic forecast in a doubly dynamical system with user inertia and information provision. Transportation Research Part C: Emerging Technologies 85, 711-731.

Lo, H.K., Luo, X., Siu, B.W., 2006. Degradable transport network: travel time budget of travelers with heterogeneous risk aversion. Transportation Research Part B: Methodological 40, 792-806. 
Lu, X., Gao, S., Ben-Elia, E., Pothering, R., 2014. Travelers' day-to-day route choice behavior with real-time information in a congested risky network. Mathematical Population Studies 21, 205-219.

Mai, T., Fosgerau, M., Frejinger, E., 2015. A nested recursive logit model for route choice analysis. Transportation Research Part B: Methodological 75, 100-112.

Martin, D., 2017. Strategic pricing with rational inattention to quality. Games and Economic Behavior 104, 131-145.

Matějka, F., 2015. Rigid pricing and rationally inattentive consumer. Journal of Economic Theory 158, 656-678.

Matejka, F., McKay, A., 2015. Rational inattention to discrete choices: A new foundation for the multinomial logit model. The American Economic Review 105, 272-298.

Paz, A., Peeta, S., 2009. Behavior-consistent real-time traffic routing under information provision. Transportation Research Part C: Emerging Technologies 17, 642-661.

Shao, H., Lam, W.H., Tam, M.L., 2006. A reliability-based stochastic traffic assignment model for network with multiple user classes under uncertainty in demand. Networks and Spatial Economics 6, 173-204.

Sims, C.A., 2003. Implications of rational inattention. Journal of monetary Economics 50, 665-690.

Sims, C.A., et al., 2010. Rational inattention and monetary economics. Handbook of Monetary Economics 3, 155-181.

Wahle, J., Bazzan, A.L.C., Klügl, F., Schreckenberg, M., 2002. The impact of real-time information in a two-route scenario using agent-based simulation. Transportation Research Part C: Emerging Technologies 10, 399-417.

Wardrop, J.G., 1952. Road paper. some theoretical aspects of road traffic research. Proceedings of the institution of civil engineers 1, 325-362. $\mathrm{Xu}, \mathrm{H}$., Zhou, J., Xu, W., 2011. A decision-making rule for modeling travelers route choice behavior based on cumulative prospect theory. Transportation Research Part C: Emerging Technologies 19, 218-228.

Yang, H., 1997. Sensitivity analysis for the elastic-demand network equilibrium problem with applications. Transportation Research Part B: Methodological 31, 55-70.

Zhang, J., Yang, H., 2015. Modeling route choice inertia in network equilibrium with heterogeneous prevailing choice sets. Transportation Research Part C: Emerging Technologies 57, 42-54. 